\title{
PARASITISMO DE OVOS DA LAGARTA-ENROLADEIRA-DA-MAÇÃ EM FUNÇÃO DO NÚMERO DE Trichogramma pretiosum RILEY (HYMENOPTERA: TRICHOGRAMMATIDAE) LIBERADO
}

\section{EGG PARASITISM OF THE BRAZILIAN APPLE LEAFROLLER IN FUNCTION OF THE NUMBER OF Trichogramma pretiosum RILEY (HYMENOPTERA: TRICHOGRAMMATIDAE) RELEASED}

\author{
Patrik Luiz PASTORI ${ }^{1}$ \\ Lino Bittencourt MONTEIRO \\ Marcos BOTTON ${ }^{3}$ \\ Alexander SOUZA ${ }^{4}$ \\ Alex Sandro POLTRONIERI ${ }^{5}$ \\ Josélia Maria SCHUBER ${ }^{5}$
}

\begin{abstract}
RESUMO
O objetivo deste trabalho foi avaliar o parasitismo diário de ovos de Bonagota salubricola (=cranaodes) (Meyrick) (Lepidoptera: Tortricidae) em função do número de Trichogramma pretiosum Riley (Hymenoptera: Trichogrammatidae) linhagem bonagota liberado na cultura da macieira. $O$ experimento foi implantado no delineamento inteiramente casualizado, em três etapas consecutivas, em pomar comercial em produção, na safra 2004/05 em Fraiburgo, SC, Brasil, constituído de cinco unidades experimentais de 1,0 ha cada, as quais receberam um dos seguintes tratamentos: ausência (testemunha), liberação de 50.000 , 100.000, 150.000 e 200.000 parasitóides ha $^{-1}$, fracionados em 50 pontos, dos quais, dois foram considerados como pontos de avaliação do parasitismo. A partir dos pontos de avaliação foram instaladas gaiolas teladas a $1 ; 4,5$ e $10 \mathrm{~m}$, à altura de $1,50 \mathrm{~m}$, que receberam adultos de $B$. salubricola. Os parasitóides foram liberados $48 \mathrm{~h}$ após a colocação dos adultos de B. salubricola, por meio de cartelas contendo ovos de Sitotroga cerealella (Oliv.) (Lepidoptera: Gelechiidae) parasitados e próximos à emergência. As folhas contendo posturas foram coletadas das gaiolas teladas aos oito, onze e cinco dias após cada momento de liberação dos parasitóides e o parasitismo avaliado após 10 dias da coleta. O parasitismo de $T$. pretiosum ajustou-se a uma função quadrática, indicando uma relação positiva entre o parasitismo e a densidade do parasitóide até 150.000 indivíduos ha-1, sendo que a partir desse número o parasitismo decresceu, evidenciando ser esta à proporção mais próxima da ideal.
\end{abstract}

Palavras-chave: Controle biológico; Bonagota salubricola; parasitóides de ovos.

\begin{abstract}
The objective of this work was to evaluate the egg parasitism of Bonagota salubricola (=cranaodes) (Meyrick) (Lepidoptera: Tortricidae) in function of the number of Trichogramma pretiosum Riley (Hymenoptera: Trichogrammatidae) strain bonagota released in apple orchards. An experiment was implanted in the experimental design entirely randomized, in three consecutive stages, in adult commercial orchard, during 2004/05 season in Fraiburgo, SC Brazil, split in five experimental units of $1.0 \mathrm{ha}$, which received one of the following treatments: absence (control), liberation of 50.000, 100.000, 150.000 and 200.000 parasitoids ha $^{-1}$, fragmented in 50 points, of which two were considered evaluation points. Starting from these points cages were installed at $1,4.5$ and $10 \mathrm{~m}$, in the height of $1.50 \mathrm{~m}$, that received adults of $B$. salubricola. The parasitoids were liberated $48 \mathrm{~h}$ after $B$. salubricola infestation in the cages, using paper cards with eggs of Sitotroga cerealella (Oliv.) (Lepidoptera: Gelechiidae) parasitized close to the emergency. The leaves containing postures were collected, eight, eleven and five days after the liberation of the parasitoids for parasitism evaluation 10 days after. The parasitism of $T$. pretiosum was adjusted to a quadratic function, indicating positive relationship between the parasitism and the density of the parasitoids up to 150.000 individuals per hectare, decreasing from this level on, evidencing to be the closest proportion of the ideal.

Key-words: Biological control; Bonagota salubricola; egg parasitoids.
\end{abstract}

\footnotetext{
'Engenheiro Agrônomo, Mestre, Doutorando do Programa de Pós-Graduação em Fitotecnia (Bolsista CNPq), Campus Universitário, Universidade Federal de Viçosa, 36.570-000, Viçosa, MG, Brasil. E-mail: plpastori@yahoo.com.br. Autor para correspondência.

${ }^{2}$ Engenheiro Agrônomo, Doutor, Professor do Departamento de Fitotecnia e Fitossanitarismo, Setor Ciências Agrárias, Juvevê, Universidade Federal do Paraná, 80.035-050, Curitiba, PR, Brasil. E-mail: Ibmonteiro@terra.com.br

${ }^{3}$ Engenheiro Agrônomo, Doutor, Pesquisador do Centro Nacional de Pesquisa Uva e Vinho, Conceição, Empresa Brasileira de Pesquisa Agropecuária, 95.700-000, Bento Gonçalves, RS, Brasil. E-mail: marcos@cnpuv.embrapa.br

${ }^{4}$ Engenheiro Agrônomo, Agrícola Fraiburgo S. A., Fraiburgo, SC, Brasil. E-mail: alexsz@agricolafraiburgo.com.br

${ }^{5}$ Engenheiro Agrônomo, Mestre, Curitiba, PR, Brasil. E-mail: alex.poltronieri@yahoo.com.br; joseliaschuber@yahoo.com.br
} 


\section{INTRODUÇÃO}

A macieira é uma das principais fruteiras de clima temperado sendo mais cultivada no Brasil e a maçã, a principal fruta exportada (MELLO, 2004). Entretanto, o cultivo é atacado por diversas pragas que causam prejuízos econômicos aos pomicultores, caso não sejam adotadas medidas de controle.

Dentre os insetos-praga destaca-se a lagarta-enroladeira-da-maçã, Bonagota salubricola (=cranaodes) (Meyrick) (Lepidoptera: Tortricidae) (KOVALESKI e RIBEIRO, 2002). As primeiras infestações foram relatadas na década de 80 (LORENZATO, 1984), quando se verificou prejuízos econômicos, principalmente sobre 'Fuji', cuja maturação é mais tardia (KOVALESKI et al., 1998). $O$ ataque do inseto pode ser observado nas folhas e nos frutos, sendo que as perdas econômicas ocorrem quando as lagartas raspam a casca dos frutos, depreciando-os comercialmente (BOTTON et al., 2000).

No âmbito da Produção Integrada de Maçãs (PIM), o manejo da lagarta-enroladeira-da-maçã é realizado apenas com os inseticidas clorpirifós e tebufenozide, o que limita as opções de ingredientes ativos para o controle do inseto (KOVALESKI e RIBEIRO, 2002), possibilitando a seleção de populações resistentes. Nesse contexto, novas alternativas de controle dessa praga devem ser investigadas, destacando-se o controle biológico, por meio da utilização de parasitóides de ovos do gênero Trichogramma Westwood (Hymenoptera: Trichogrammatidae). Esses agentes de controle biológico possuem ampla distribuição geográfica e são altamente especializados e eficientes (PRATISSOLI e PARRA, 2001; HAJI et al., 2002; PRATISSOLI et al., 2003).

Trichogramma pretiosum (Riley) (Hymenoptera: Trichogrammatidae) foi observado parasitando naturalmente ovos de $B$. salubricola, em pomares de macieiras na região de Fraiburgo, SC (MONTEIRO et al., 2004), abrindo perspectivas para a utilização desse parasitóide em programas de controle biológico aplicado. Entretanto, o sucesso desses programas depende basicamente de pesquisas que avaliem os fatores que afetam a eficiência dos parasitóides no campo, tais como a capacidade de busca de hospedeiros (PRATISSOLI et al., 2005), o número de insetos liberados (BOTELHO, 1997), a densidade da praga, a espécie ou a linhagem de Trichogramma liberada, a época e o número de liberações, o método de distribuição, a fenologia da cultura, o número de outros inimigos naturais presentes e as condições climáticas vigentes quando da liberação (SMITH et al., 1986).

Dentro deste contexto, o objetivo deste trabalho foi avaliar o parasitismo diário de ovos de Bonagota salubricola (=cranaodes) (Meyrick) (Lepidoptera: Tortricidae) em função do número de Trichogramma pretiosum Riley (Hymenoptera: Trichogrammatidae) linhagem bonagota liberado em pomares comerciais de macieiras em produção.

\section{MATERIAL E MÉTODOS}

O experimento foi conduzido em três etapas, adotando o delineamento experimental inteiramente casualizado, na safra 2004/05, em um pomar comercial de macieiras (Malus domestica Borkh.) em produção, plantado em 1990, no espaçamento de $1,5 \times 4,5 \mathrm{~m}$ (plantas $\mathrm{x}$ linhas) com altura de plantas entre 3,0 a 3,5 m, pertencente à empresa Agrícola Fraiburgo S.A., localizado no município de Fraiburgo, $\mathrm{SC}$, Brasil $\left(27^{\circ} 02^{\prime} \mathrm{S}\right.$ e $\left.50^{\circ} 55^{\prime} \mathrm{W}\right)$. Cada etapa constou de um momento diferente de liberação dos parasitóides, sendo a seqüência metodológica repetida em cada etapa, com intervalo de sete dias, para que não houvesse interferência da liberação anterior. O pomar foi estabelecido numa combinação de onze plantas de 'Gala' (produtora) para uma de 'Fuji' (polinizadora) em cada linha de plantio sucessivamente.

Para a estimativa do número de T. pretiosum a ser liberado no campo, em março de 2005, após a colheita da 'Gala', foram delimitadas cinco unidades experimentais (UE's) de 1,0 ha cada, que receberam os seguintes tratamentos: ausência de liberação (testemunha), liberação de 50.000, 100.000, 150.000 e 200.000 parasitóides ha-1 (T. pretiosum linhagem bonagota), fracionados em 50 pontos de liberação em cada UE, distanciados de $20 \mathrm{~m}$ nas linhas e $9 \mathrm{~m}$ entre linhas de forma alternada (Figura 1). Dois destes pontos foram definidos, ao acaso, como pontos de avaliação do parasitismo.

A partir dos pontos de avaliação do parasitismo foram instaladas gaiolas de madeira de $50 \times 40 \times 40 \mathrm{~cm}$, envoltas por tule malha de 6,0 $\mathrm{mm}^{2}$, de cor verde e fixadas nas macieiras à altura de 1,50 a $1,70 \mathrm{~m}$ acima da superfície do solo, no sentido das linhas de plantio e distanciadas de 1; 4,5 e $10 \mathrm{~m}$ (Figura 1). Na linha de plantio subseqüente foram instaladas mais três gaiolas, nas mesmas distâncias em relação ao ponto de liberação, mas no sentido contrário ao realizado na linha do ponto de liberação (Figura 1). Cada gaiola recebeu cinco fêmeas e três machos de $B$. salubricola, criados no Laboratório de Manejo Integrado de Pragas (LMIP) da Universidade Federal do Paraná, segundo metodologia desenvolvida por PARRA et al. (1995). No interior das gaiolas foram introduzidos ramos de macieira, sem destacar das plantas, para que as mariposas fizessem posturas nas folhas. Como fonte de alimento, foi aspergido solução de mel a $10 \%$ em algumas folhas dentro da gaiola. Na área testemunha, as gaiolas foram instaladas ao acaso nas mesmas distâncias definidas para os tratamentos.

T. pretiosum linhagem bonagota, foi coletado em ovos de $B$. salubricola, em plantios comerciais de macieira em Fraiburgo, SC (MONTEIRO et al., 2004), mantido e multiplicado utilizando o hospedeiro alternativo Sitotroga cereallela (Oliv.), (Lepidoptera: Gelechiidae), no laboratório da Associação de Fruticultores de Fraiburgo (AFF), Fraiburgo, SC, Brasil, obedecendo à metodologia desenvolvida por NAVARRO (1998). 


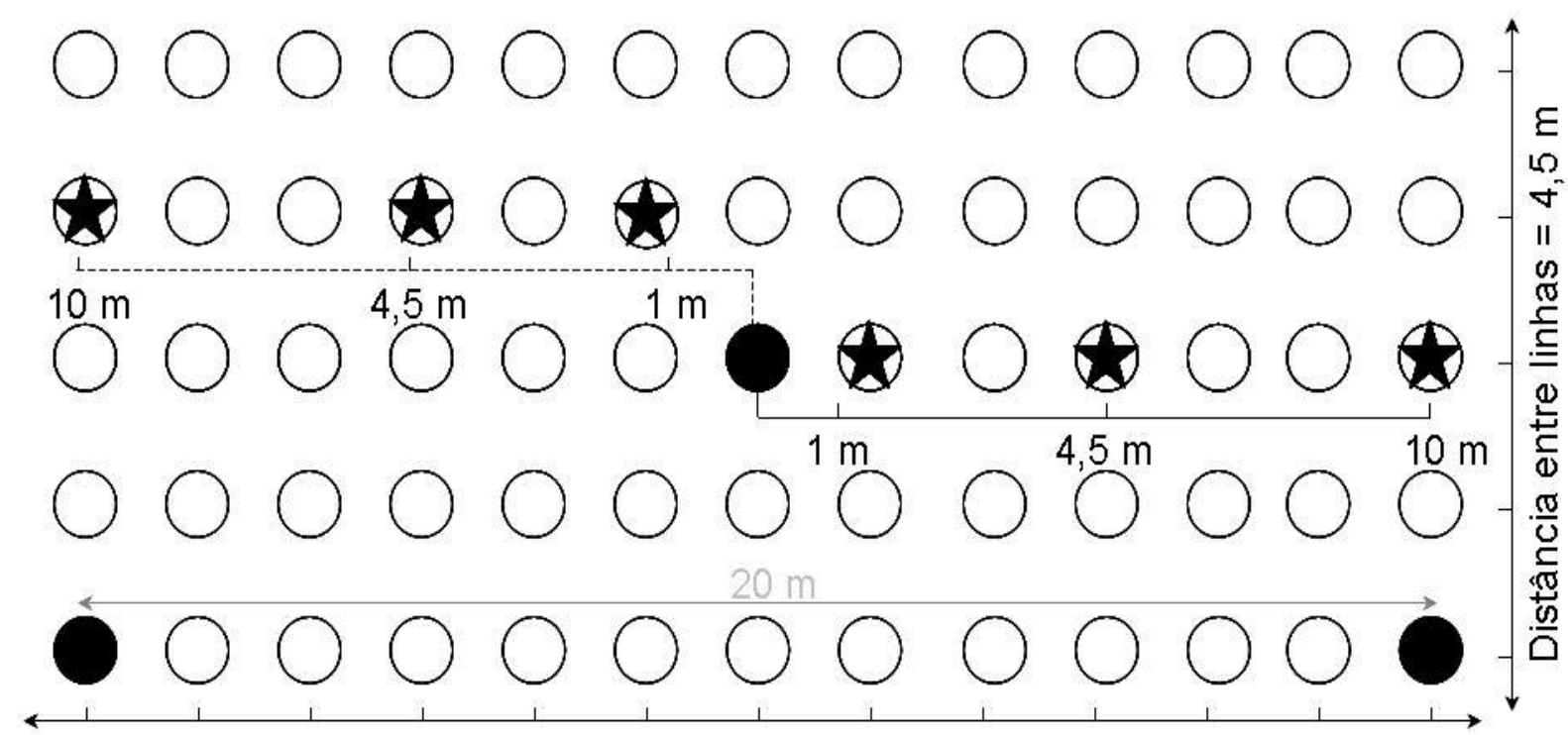

Distância entre plantas $=1,5 \mathrm{~m}$

Plantas
- Plantas abrigando pontos de liberação de Trichogramma
Plantas abrigando pontos de avaliação do parasitismo

FIGURA 1 - Distribuição dos pontos de liberação de Trichogramma pretiosum Riley (Hymenoptera: Trichogrammatidae) linhagem bonagota e dos pontos de avaliação do parasitismo [gaiolas de madeira abrigando adultos de Bonagota salubricola (Meyrick) (Lepidoptera: Tortricidae)] posicionadas em diferentes distâncias, nas linhas de plantio e linhas subseqüentes, em pomar comercial de macieira. Fraiburgo, SC, Brasil. 2005.

Os parasitóides foram liberados no pomar $48 \mathrm{~h}$ após a colocação de $B$. salubricola nas gaiolas, por meio de cartelas contendo ovos de S. cerealella parasitados, próximo à emergência dos adultos. As cartelas foram fixadas nas macieiras à altura de 1,70 $\mathrm{m}$ da superfície do solo e protegidas por uma tela de náilon branco, no formato de envelope, visando evitar predadores.

Em cada ponto de avaliação do parasitismo, folhas de macieira contendo as posturas de $B$. salubricola foram coletadas nas gaiolas, aos oito dias após a liberação de $T$. pretiosum na primeira etapa; aos onze dias após a liberação na segunda etapa; e aos cinco dias após a liberação na terceira etapa. O parasitismo foi avaliado após 10 dias de cada coleta por meio da contagem de ovos escuros, utilizando um microscópio estereoscópico com aumento de 10 vezes.

Os resultados de cada etapa foram transformados em percentagem de parasitismo diário e submetidos à análise de regressão.

\section{RESULTADOS E DISCUSSÃO}

As curvas de parasitismo médio diário de $T$. pretiosum linhagem bonagota, em ovos de $B$. salubricola, ajustaram-se a uma função quadrática (Figuras 2 e 3 ).
O parasitismo de ovos de $B$. salubricola na linha subseqüente àquela do ponto de liberação foi semelhante ao observado nas linhas com pontos de liberação, o que permite inferir que o parasitóide apresentou boa distribuição radial na área de estudo.

A porcentagem de parasitismo foi proporcional à densidade do parasitóide, obtendose maior percentual de parasitismo com o aumento do número de parasitóides liberados, até próximo de 150.000 indivíduos por hectare; a partir deste número, o parasitismo decresceu (Figuras 2 e 3). De acordo com KNIPLING (1979), com o aumento no número de parasitóides por ovo da praga alvo, há uma tendência de redução na eficiência do parasitóide, decorrente da menor probabilidade de um indivíduo encontrar ovos não parasitados, podendo ocorrer, em muitos casos, superparasitismo. Assim, se for liberada grande quantidade de parasitóides e a densidade do hospedeiro for baixa, ocorrerá uma baixa taxa de parasitismo (NEIL e SPECHT, 1990), situação esta que talvez explique as taxas de parasitismo encontradas neste estudo. Além disso, é possível que a redução do desempenho da linhagem no campo, seja devido à presença de ovos de outros lepidópteros das famílias Noctuidae e Geometridae (FONSECA, 2006), que poderiam ser preferidos pelas fêmeas de $T$. pretiosum ou então, em função da camada de secreção sobre os ovos de $B$. salubricola que dificulta o parasitismo (BASSO et al., 1998). 
PASTORI, P. L. et al. Parasitismo de ovos...
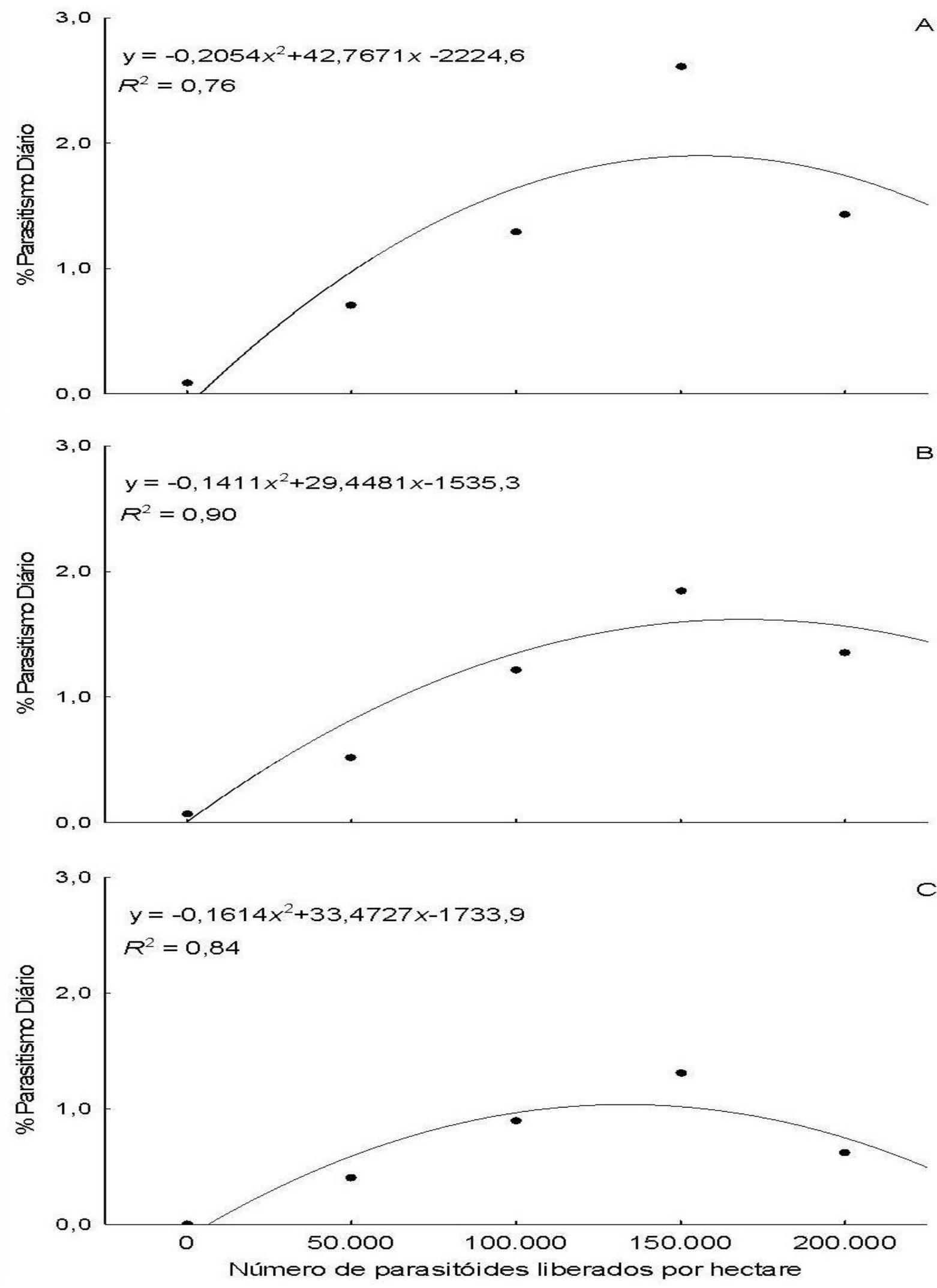

FIGURA 2 - Relação entre o número de Trichogramma pretiosum linhagem bonagota liberado por hectare e o parasitismo diário em ovos de Bonagota salubricola (=cranaodes) (Meyrick) (Lepidoptera: Tortricidae) em gaiolas posicionadas em linhas, distanciadas em: $A=1 \mathrm{~m}, \mathrm{~B}=4,5 \mathrm{~m}$ e $\mathrm{C}=10 \mathrm{~m}$ do ponto de liberação. Fraiburgo, SC, Brasil. 2005. 

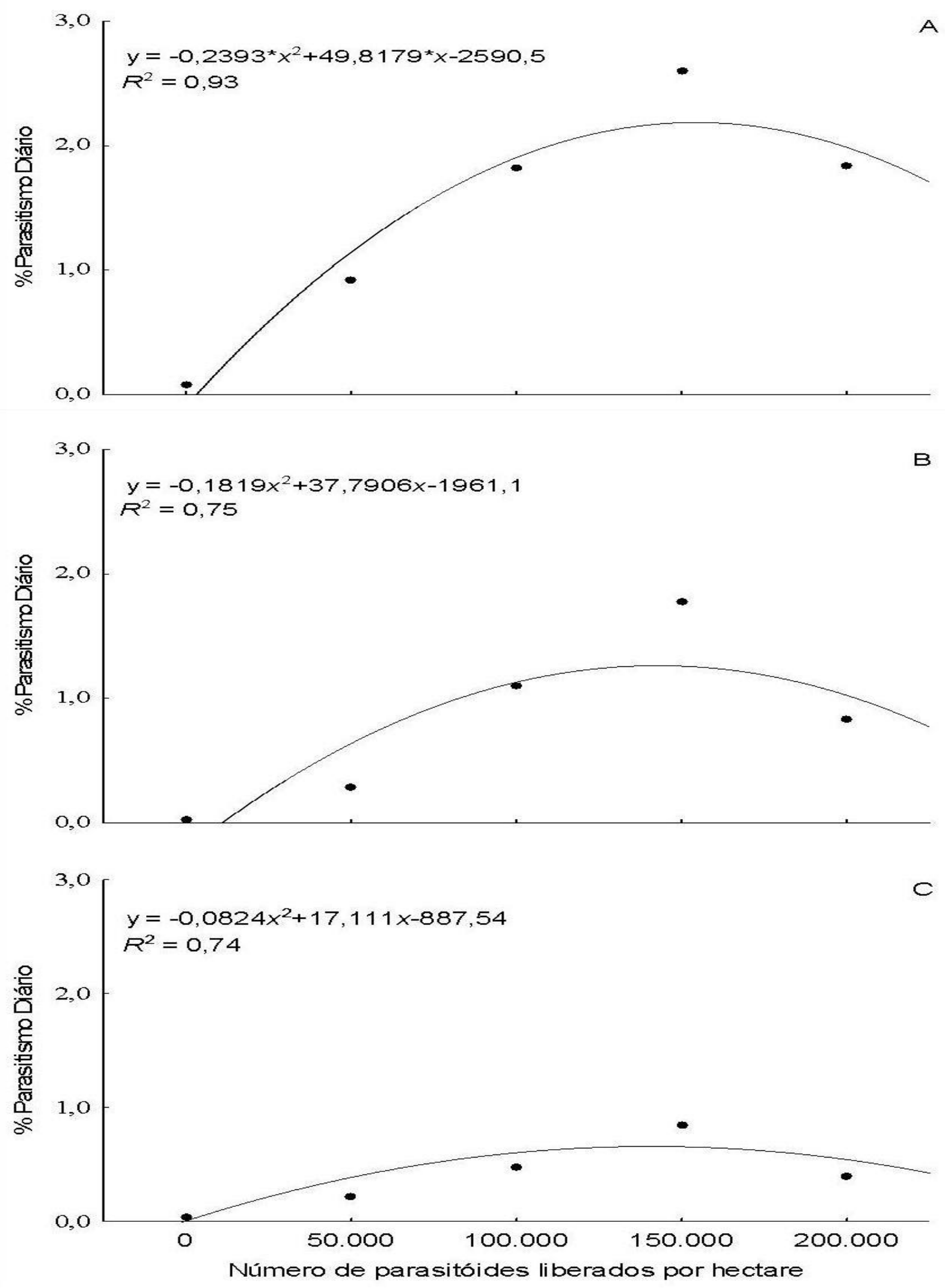

FIGURA 3 - Relação entre o número de Trichogramma pretiosum linhagem bonagota liberado por hectare e o parasitismo diário em ovos de Bonagota salubricola (=cranaodes) (Meyrick) (Lepidoptera: Tortricidae) em gaiolas posicionadas em linhas subseqüentes, distanciadas em: $A=1 \mathrm{~m}, \mathrm{~B}=4,5 \mathrm{~m}$ e $\mathrm{C}=10$ $\mathrm{m}$ do ponto de liberação. Fraiburgo, SC, Brasil. 2005. 
Durante o período de realização do experimento, não foram aplicados produtos fitossanitários no pomar, a temperatura máxima média foi de $27,1^{\circ} \mathrm{C}$ e a mínima média de $15,4{ }^{\circ} \mathrm{C}$ e não ocorreu geada, chuva ou granizo, condições que, a princípio, influenciariam negativamente a atividade dos parasitóides.

No caso da fruticultura, poucos estudos foram conduzidos no sentido de se determinar a quantidade de parasitóides a serem liberados e temse recomendado liberações que variam de 70.000 a 3,8 milhões de parasitóides ha $^{-1}$ (GLENN e HOFFMANN, 1997; MILLS et al., 2000). O número de parasitóides a ser liberado é variável em função da fenologia da planta, da espécie e linhagem de Trichogramma, bem como da dinâmica de postura do hospedeiro e condições climáticas (BOTELHO, 1997; PRATISSOLI et al., 2005).

Variações no número de parasitóides liberados podem ser observadas em trabalhos de diversos autores para culturas anuais. BROGLIOMICHELETTI et al. (2007) observaram parasitismo ideal por Trichogramma galloi Zucchi (Hymenoptera: Trichogrammatidae) com liberações de 150.000 a 175.000 parasitóides ha $^{-1}$, visando o controle de Diatraea saccharalis (Fabricius) (Lepidoptera: Crambidae) em cana-de-açúcar. NOGUEIRA de SÁ e PARRA (1993) demonstraram que, para Helicoverpa zea (Boddie) (Lepidoptera: Noctuidae) em milho, três liberações de 100.000 adultos ha-1 de $T$. pretiosum durante o ciclo da cultura, promovem eficiente controle dessa praga. Entretanto no Chile, VELÁSQUEZ e GERDING (2006) trabalhando com Trichogramma nerudai Pintureau, Gerding e Cysternas (Hymenoptera: Trichogrammatidae), observaram $20 \%$ de redução de danos por $H$. zea com liberações de 1.600 .000 parasitóides ha ${ }^{-1}$. NAVARRO (1998) obteve resultados satisfatórios com 10 liberações de 200.000 adultos ha-1 de $T$. pretiosum, para o controle de Tuta absoluta (Meyrick) (Lepidoptera: Gelechiidae) e Neoleucinodes elegantalis (Guenée) (Lepidoptera: Crambidae) em tomateiro, número este, corroborado por HAJI et al. (2002) em tomateiro industrial na região do Submédio do São Francisco.

Para o controle de Heliothis spp. em algodoeiro, liberações de 247.000 parasitóides por hectare resultaram em valores de 24 a $73 \%$ de parasitismo (RIDGWAY et al., 1977), enquanto STINNER et al. (1974) obtiveram em média, $51 \%$ de parasitismo para liberações de até $957.000 \mathrm{mil}$ adultos ha ${ }^{-1}$ de Trichogramma. Segundo NOGUEIRA de SÁ e PARRA (1993), o número ideal de parasitóide a ser liberado também pode mudar de acordo com a densidade de plantio e da intensidade da infestação da praga no campo. Além disso, as espécies de Trichogramma apresentam grande variação no seu comportamento de procura, preferência hospedeira e resposta às condições ambientais (HASSAN, 1997).
A estreita relação entre parasitóide/ hospedeiro também pode ser verificada nos estudos conduzidos por PRATISSOLI et al. (2005) e NAVA e PARRA (2006), que trabalhando com $T$. pretiosum em ovos de $T$. absoluta e Stenoma catenifer Walsingham (Lepidoptera: Elachistidae), obtiveram a relação 16 e 20 a 30 parasitóides por ovo, respectivamente. Neste sentido, GARCIA (1998) trabalhando com $T$. pretiosum em ovos de Ecdytolopha aurantiana (Lima) (Lepidoptera: Tortricidae), verificou que o aumento nas taxas de parasitismo deste hospedeiro poderiam ser obtidas se fossem conduzidos estudos com relações superiores a 12,8 parasitóides por ovo.

Estudos visando a utilização de T. pretiosum para o controle de $B$. salubricola foram conduzidos em laboratório (FONSECA et al., 2005; BORBA et al., 2006; PASTORI et al., 2007). Entretanto NAVARRO (1998) relata que, para se obter o número de parasitóides a ser liberado no campo, não bastam experiências obtidas de ensaios, mas do conhecimento apurado das relações parasitóide/ praga/cultivo. Neste sentido, de acordo com os resultados obtidos, a proporção de 150.000 parasitóides ha-1 apresentou a maior porcentagem de parasitismo diário sendo recomendada para liberação em plantios comerciais de macieiras. Entretanto, o controle biológico utilizando $T$. pretiosum deve ser considerado uma ferramenta para o manejo integrado da lagarta-enroladeira-damaçã e outras medidas devem ser sincronizadas para que o controle seja efetivo. Além disso, estudos devem ser conduzidos para se determinar a distribuição espacial, bem como conhecer o comportamento e o período crítico de oviposição da praga na cultura, além de identificar o período fenológico da planta mais susceptível ao ataque da praga.

\section{CONCLUSÃO}

A liberação de 150.000 parasitóides ha-1 (Trichogramma pretiosum linhagem bonagota) resulta na maior porcentagem de parasitismo diário de ovos de Bonagota salubricola em pomares de macieira.

\section{AGRADECIMENTOS}

Ao Conselho Nacional de Desenvolvimento Científico e Tecnológico (CNPq) e à Coordenação de Aperfeiçoamento de Pessoal de Nível Superior (CAPES), pela concessão das bolsas de estudo, a Universidade Federal do Paraná, a Empresa Agrícola Fraiburgo S.A. e a Associação de Fruticultores de Fraiburgo, por possibilitarem a execução da presente pesquisa. 


\section{REFERÊNCIAS}

1. BASSO, C.; GRILLE, G.; POMPANON, F.; ALLEMAND, R.; PINTUREAU, B. Comparación de los caracteres biológicos y etológicos de Trichogramma pretiosum y de T. exiguum (Hymenoptera: Trichogrammatidae). Revista Chilena de Entomologia, v. 25, n. 1, p. 45-53, 1998.

2. BORBA, R. S.; GARCIA, M. S.; KOVALESKI, A.; COMIOTTO, A.; CARDOSO, R. L. Biologia e exigências térmicas de Trichogramma pretiosum Riley (Hymenoptera: Trichogrammatidae) sobre ovos de Bonagota cranaodes (Meyrick) (Lepidoptera: Tortricidae). Ciência Rural, v. 36, n. 5, p.1345-1352, 2006.

3. BOTELHO, P. M. Eficiência de Trichogramma em campo. In: PARRA, J. R. P.; ZUCCHI, R. A. (Eds.). Trichogramma e o controle biológico aplicado. Piracicaba: FEALQ, 1997. p. 303-318.

4. BOTTON, M.; NAKANO, O.; KOVALESKI, A. Controle químico da lagarta-enroladeira Bonagota cranaodes (Meyrick) na cultura da macieira. Pesquisa Agropecuaria Brasileira, v. 35, n. 11, p. 2139-2144, 2000.

5. BROGLIO-MICHELETTI, S. M. F.; PEREIRA-BARROS, J. L.; SANTOS, A. J. N.; CARVALHO, L. W. T.; CARVALHO, L. H. T.; OLIVEIRA, C. J. T. Efeito do número de adultos de Trichogramma galloi Zucchi, 1988 (Hymenoptera: Trichogrammatidae) liberados em semanas sucessivas, para o controle de Diatraea saccharalis (Fabricius, 1794) (Lepidoptera: Crambidae). Ciência e Agrotecnologia, v. 31, n. 1, p. 53-58, 2007.

6. FONSECA, F. L. da. Ocorrência, monitoramento, caracterização de danos e parasitismo de Noctuidae e Geometridae em pomares comerciais de macieira em Vacaria, Rio Grande do Sul, Brasil. 97 f. Tese (Doutorado em Entomologia) - Curso de Pós-Graduação em Ciências Biológicas - Entomologia, Universidade Federal do Paraná, Curitiba, 2006

7. FONSECA, F. L. da.; KOVALESKI, A.; FORESTI, J.; RINGENBERG, R. Desenvolvimento e exigências térmicas de Trichogramma pretiosum Riley (Hymenoptera: Trichogrammatidae) em ovos de Bonagota cranaodes (Meyrick) (Lepidoptera: Tortricidae). Neotropical Entomology, v. 34, n. 6, p. 945-949, 2005.

8. GARCIA, M. S. Bioecologia e potencial de controle biológico de Ecdytelopha aurantiana (Lima, 1927) (Lepidoptera: Tortricidae), o bicho-furão-dos-citrus, através de Trichogramma pretiosum Riley, 1879 (Hymenoptera: Trichogrammatidae). 118 f. Tese (Doutorado em Entomologia) - Curso de Pós-Graduação em Entomologia, Universidade de São Paulo/Escola Superior de Agricultura "Luiz de Queiroz", Piracicaba, 1998.

9. GLENN, D. C.; HOFFMANN, A. A. Developing a commercially viable system for biological control of light brown apple moth (Lepidoptera: Tortricidae) in grapes using endemic Trichogramma (Hymenoptera: Trichogrammatidae). Journal of Economic Entomology, v. 90, n. 2, p. 370-382, 1997.

10. HAJI, F. N. P.; PREZOTTI, L.; CARNEIRO, J. S.; ALENCAR, J. A. Trichogramma pretiosum para o controle de pragas no tomateiro industrial. In: PARRA, J. R. P.; BOTELHO, P. S. M.; CORRÊA-FERREIRA, B. S.; BENTO, J. M. S. (Eds.). Controle biológico no Brasil: parasitóides e predadores. Piracicaba: Manole, 2002. p. 477-494.

11. HASSAN, S. A. Seleção de espécies de Trichogramma para o uso em programas de controle biológico. In: PARRA, J. R. P.; ZUCCHI, R. A. (Eds.). Trichogramma e o controle biológico aplicado. Piracicaba: FEALQ, 1997. p. 183-205.

12. KNIPLING, E. F. Insect population suppression by augmenting the number of parasites and predators in crop ecossystems. In: KNIPLING, E. F (Ed.). The basic principles of insect populations suppression and management. Washington: USDA, 1979. p. 135-197. (Agriculture Handbook, 512).

13. KOVALESKI, A.; BOTTON, M.; EIRAS, A. E.; VILELA, E. F. Lagarta-enroladeira da macieira: bioecologia e controle. Bento Gonçalves: Embrapa CNPUV, 1998. 22 p. (Circular Técnica, 24).

14. KOVALESKI, A.; RIBEIRO, L. G. Manejo de pragas na produção integrada de maçã. Bento Gonçalves: Embrapa CNPUV, 2002. 15 p. (Circular Técnica, 34).

15. LORENZATO, D. Ensaio laboratorial de controle da "traça-da-maçã" Phtheochroa cranaodes Meyrick, 1937 com Bacillus thuringiensis Berliner e inseticidas químicos. Agronomia Sulriograndense, v. 20, n. 1, p. 157-163, 1984.

16. MELLO, L. M. R. Produção e mercado brasileiro de maçã. Bento Gonçalves: Embrapa CNPUV, 2004. 4 p. (Comunicado Técnico, 50).

17. MILLS, N.; PICKEL, C.; MASFIELD, S.; MCDOUGALL, S.; BUCHNER, R.; CAPRILE, J.; EDSTRON, J.; ELKINS, R.; HASEY, J.; KELLEY, K.; KRUEGER, B.; OLSON, B.; STOCKER, R. Mass releases of Trichogramma wasps can reduce damage from codling moth. California Agriculturae, v. 56, n. 6, p. 22-25, 2000.

18. MONTEIRO, L. B.; SOUZA, A.; BELLI, E. L.; SILVA, R. B. Q.; ZUCCHI, R. A. Ocorrência de Trichogramma pretiosum Riley (Hymenoptera: Trichogrammatidae) em ovos de Bonagota cranaodes (Meyrick) (Lepidoptera: Tortricidae) em macieira. Revista Brasileira de Fruticultura, v. 26, n. 1, p. 171-172, 2004.

19. NAVA, D. E.; PARRA, J. R. P. Determinação do número e da idade de ovos de Stenoma catenifer no parasitismo de Trichogramma pretiosum. Revista de Agricultura, v. 81, n. 1, p. 71-80, 2006.

20. NAVARRO, M. A. Trichogramma spp. Produción, uso y manejo en Colombia. Valle del Cauca: Guadalajara de Buga, 1998, $176 \mathrm{p}$.

21. NEIL, K.; SPECHT, A. Field releases of Trichogramma pretiosum Riley (Hymenoptera: Trichogrammatidae) for suppression of corn earworn, Heliothis zea (Bodie) (Lepidoptera: Noctuidae), egg populations on sweet corn in Nova Scotia. Canadian Entomology, v. 122, n. 3, p. 1259-1266, 1990.

22. NOGUEIRA de SÁ, L. A.; PARRA, J. R. P. Efeito do número e intervalo entre liberações de Trichogramma pretiosum Riley no parasitismo e controle de Helicoverpa zea (Boddie), em milho. Scientia Agricola, v. 50, n. 2, p. 355-359, 1993.

23. PARRA, J. R. P.; EIRAS, A. E.; HADDAD, M. L.; VILELA, E. F.; KOVALESKI, A. Técnica de criação de Phtheochroa cranaodes Meyrick (Lepidoptera: Tortricidae) em dieta artificial. Revista Brasileira de Biologia, v. 55, n. 4, p. 537-543, 1995.

24. PASTORI, P. L.; MONTEIRO, L. B.; BOTTON, M.; PRATISSOLI, D. Capacidade de parasitismo de Trichogramma pretiosum Riley (Hymenoptera: Trichogrammatidae) em ovos de Bonagota salubricola (Meyrick) (Lepidoptera: Tortricidae) sob diferentes temperaturas. Neotropical Entomology, v. 36, n. 6, p. 926-931, 2007. 
PASTORI, P. L. et al. Parasitismo de ovos...

25. PRATISSOLI, D.; PARRA, J. R. P. Seleção de linhagens de Trichogramma pretiosum Riley (Hymenoptera: Trichogrammatidae) para o controle das traças Tuta absoluta (Meyrick) e Phthorimaea operculella (Zeller) (Lepidoptera: Gelechiidae). Neotropical Entomology, v. 30, n. 2, p. 277-282, 2001.

26. PRATISSOLI, D.; FORNAZIER, M. J.; HOLTZ, A. M.; GONÇALVES, J. R.; CHIORAMITAL, A. B.; ZAGO, H. B. Ocorrência de Trichogramma pretiosum em áreas comerciais de tomate, no Espírito Santo, em regiões de diferentes altitudes. Horticultura Brasileira, v. 21, n. 1, p. 73-76, 2003.

27. PRATISSOLI, D.; THULER, R. T.; ANDRADE, G. S.; ZANOTTI, L. C. M.; SILVA, A. F. Estimativa de Trichogramma pretiosum para o controle de Tuta absoluta em tomateiro estaqueado. Pesquisa Agropecuária Brasileira, v. 40, n. 7, p. 715-718, 2005.

28. RIDGWAY, R. L.; KING, E. G.; CARRILLO, J. L. Argumentation of natural enenies for control of plant pests in the Wetern Hemisphere. In: RIDGWAY, R. L.; VINSON, S. B. (Eds.). Biological control by argumentation of natural enenies. New York: Plenum Press, 1977. p. 379-416.

29. SMITH, S. M.; HUBBES, M.; CARROW, J. R. Factors affecting inundative releases of Trichogramma minutum Ril. against the spruce budworm. Journal of Applied Entomology, v. 101, n. 1, p. 29-39, 1986.

30. STINNER, R. E.; RIDGWAY, R. L.; COPEDGE, J. R.; MORRISON, R. K.; DICKERSON Jr., W. A. Parasitism of Heliothis eggs after field releases of Trichogramma pretiosum, in cotton. Environmental Entomology, v. 3, n. 4, p. 497-500. 1974.

31. VELÁSQUEZ, F. C.; GERDING P. M. Evaluación de diferentes especies de Trichogramma spp. para el control de Helicoverpa zea (Boddie) (Lepidoptera: Noctuidae). Agricultura Técnica, v. 66, n. 4, p. 411-415, 2006.

Recebido em 14/02/2008

Aceito em 01/07/2008 
